Malischnig, D., Griffiths, M.D. \& Auer, M. (2018). A brief overview of the Austrian gambling regulation and implications for player protection and harm minimization. Gaming Law Review, 22, 564-567.

\title{
A brief overview of the Austrian gambling regulation and implications for player protection and harm minimisation
}

\section{Doris Malischnig', Mark D. Griffiths², Michael Auer ${ }^{3}$}

1 Institute for Addiction Prevention Vienna, Modecenterstrasse 14, 1030 Vienna, Austria

2 International Gaming Research Unit, Psychology Department, Nottingham Trent University, Burton Street, Nottingham, NG1 4FQ

3 neccton 1td, 207 Regent Street, London, W1B 3HH

Dr. Doris Malischnig studied Psychology at the University of Vienna and Bremen. She is working as a Project Manager for E-Mental Health issues for the Institute for Addiction Prevention of the Office of Addiction and Drug Policy of Vienna and she is lecturing on a university level in Austria about gambling prevention. Doris has been a regular speaker on national and international professional congresses.

Dr. Mark Griffiths is a Chartered Psychologist and Distinguished Professor of Behavioural Addiction at the Nottingham Trent University, and Director of the International Gaming Research Unit. He is internationally known for his work on gambling and gaming addictions has published over 760 refereed research papers, five books, 150+ book chapters and over 1000 other articles. He has won 19 national and international awards for his work. He also does a lot of freelance journalism and has appeared on over 3000 radio and television programs, and written over 350 articles for national and international newspapers and magazines.

Dr. Michael Auer is a Psychologist and a Statistician and he received his $\mathrm{PhD}$ in Psychology from Nottingham Trent University. He is a director at neccton and has first authorship on more behavioural tracking publications in the area of gambling than anybody else. Apart from that he is also a regular speaker at conferences and regulatory symposiums throughout 
In most countries, gambling is subject to specific regulations. There appears to be a relationship between the total number of types of gambling that are legal in a state and the gambling involvement of state residents ${ }^{1}$. Most frequently, regulations are organized into three categories: (i) restrictions on the general availability of gambling; (ii) restrictions on who can gamble; and (iii) restrictions on how gambling is provided ${ }^{2}$. In recent years, there has been a strong shift in gambling regulations across Europe. Many European regulations mostly refer to land-based gambling. In some countries (e.g., Germany) online gambling is not legally allowed. Nonetheless, in recent years internet gambling has become one of the fastest growing forms of gambling ${ }^{3}$. It is growing rapidly in terms of popularity and products offered, and is easily accessible ${ }^{4}$.

Austria is an EU member state in central Europe with a long history in gambling. In 1751, a lottery with 90 numbers with the official name "Lotto di Genova" was introduced and the first casinos opened close to Vienna in 1934. Data on gambling participation, the severity of gambling-related problems, and the acceptance of measures for the protection of gamblers were collected in Austria for the first time in 2009, some of which were replicated by Kalke et al. in $2015^{5}$. The prevalence rate of individuals with problematic gambling behaviour in Austria was $1.1 \%$ in both 2009 and in 2015. This demonstrates that Austria is in the lowerthird of problem gambling prevalence rates compared to other countries in Europe ${ }^{6}$. This brief paper provides an overview of current Austrian gambling regulations and the implications for player protection and harm minimisation. We believe that this is important because the Austrian gambling regulation includes a few specific peculiarities that are not widely known due to the lack of English speaking documentation or academic studies.

\section{National regulation}

\footnotetext{
${ }^{1}$ Welte, J.W., Tidwell, M.C., Barnes, G.M., Hoffman, J.H., Wieczorek, W.F. (2016). The relationship between the number of types of legal gambling and the rates of gambling behaviors and problems across U.S. States. Journal of Gambling Studies 32: 379-390

2 Williams, R.J., West, B.L., \& Simpson, R.I. (2012). Prevention of Problem Gambling: A Comprehensive Review of the Evidence, and Identified Best Practices. Report prepared for the Ontario Problem Gambling Research Centre and the Ontario Ministry of Health and Long Term Care. October 1, 2012. http://hdl.handle.net/10133/3121

${ }^{3}$ Gainsbury, S. (2012). Internet gambling. Current research findings and implications. New York: Springer.

${ }^{4}$ Lycka, M. Online gambling: Towards a transnational regulation. Gaming Law Rev Econ. 2011;15(4):179-195.

${ }^{5}$ Glücksspielverhalten der österreichischen Bevölkerung und ihre Akzeptanz von Spielerschutzmaßnahmen Ergebnisse der Repräsentativbefragungen 2009 und 2015. (Gambling Behaviour and Acceptance of Protection Measures for Gamblers in the Austrian General Population - Results of the Representative Surveys in 2009 and 2015)

${ }^{6}$ Calado, F \& Griffiths, M. (2016). Problem gambling worldwide: An update and systematic review of empirical research (2000-2015). Journal of Behavioral Addictions 5(4), 592-613.
} 
Austria comprises nine federal states and some types of gambling are subject to national regulation (e.g., licences for casinos, lottery products, video lottery terminals, and online gambling) while other types are subject to the federal states regulation (e.g., licence for slot machines and sports betting). In principle, gambling in Austria is regulated by the national gambling law "Glücksspielgesetz" (GSpG) dating from November 28, 1989. The GSpG defines gambling as games in which the outcome is completely or mostly determined by chance. The GSpG says that the state reserves the right to conduct games of chance. The Austrian state is the only legal body with the authority to grant casino, lottery, and poker licenses after a public tender. The objective of the Austrian monopoly and license system is to minimize gambling-related harm and crime issues while providing sufficient gambling opportunities for those who want to gamble. The licensees are monitored by the Ministry of Finance which - among others - evaluates gambling advertising and its use in promoting legal gambling products.

\section{Lottery licenses}

The national lottery license is awarded for a period of 15 years. This concession also includes the right to offer online gambling and video lottery terminals (VLTs). The license is awarded after a tendering process for the aforementioned 15 years. On October 10, 2011, Austrian Lotteries was granted a license to offer lottery games (instant games as well as draw-based games) and is valid from October 1, 2012 until September 30, 2027. Austrian Lotteries is the only lottery licensee in Austria and the license includes the permission to offer lottery products via sales outlets in Austria and to offer online gambling (for all types of games of chance, including lottery tickets, scratch cards, casino games and poker) as well as VLTs. Austrian Lotteries has the right to operate 5000 VLTs in Austria under the October 2011 lottery license. On VLTs, the odds of winning and the pay-out rates are fixed and approved by the Federal Ministry of Finance. The decision concerning whether somebody wins or loses is not made by the gambling device itself, but by a central random number generator in the computing centre of Austrian Lotteries. Online gambling is operated by win2day, a $100 \%$ subsidiary of Austrian Lotteries. win2day is, therefore, the only Austrian-licensed operator of games of chance on the internet. win2day offers lottery (draw-based and instant) games and casino games as well as poker and sports betting. In Austria, sports betting is not regarded as a game of chance.

\section{Casino licenses}


On December 19, 2012, Casinos Austria received six casino licenses for urban regions. Six more licenses for rural regions were granted to Casinos Austria on September 9, 2013. Casinos Austria continued to operate their casinos as before. To date, all court cases have confirmed the adherence of Austrian gambling law within EU law. Rural and urban casino licenses are valid until 31 December, 2027, and 31 December, 2030, respectively.

\section{Player protection requirements by state and technical inspection}

The Austrian Ministry of Finance represents the gambling authority. The GSpG also describes specific requirements for the licensees which are monitored by the Austrian Ministry of Finance. A specific department for player protection was established in the Ministry of Finance. The duties of the department are:

- Coordination of the cooperation between governmental bodies and non-governmental organizations with respect to player protection on regional, federal, national, and international level.

- Representation of player protection including gambling addiction prevention in Austria.

- The professional assessment of player protection concepts of the federal concessionaires.

- Advisory role and support of the gambling authority.

- Information about gambling-related risks.

- Support of gambling research and help organizations.

- Information about problem gambling support helplines.

As required by GSpG ( $(25)$, the frequency of casino visits and the gambling intensity must be monitored by the licensee. The same goes for operators of VLT outlets (GSpG §12a). The licensee is obligated to act if there is an indication that visitors spend more than they can afford. If there is a reasonable belief that the person can no longer afford to gamble, the operator has the right to obtain information about the individual's income. The operator can negotiate a maximum number of visits or exclude the visitor for a specific amount of time. Visitors can also exclude themselves from casinos permanently or temporarily. All slot machines in the 12 casinos and all VLTs are connected to the Austrian computing centre (Bundesrechenzentrum), which is a $100 \%$ subsidiary of the Austrian Ministry of Finance. They deliver daily information about events (e.g., if a slot machine has been opened) and 
monetary information (such as the amount of money inserted). This happens via the G2S protocol and is required by the GSpG. However, visitors must be of age, which means for Austria, that a minimum age of 18 years is required to enter a casino or a VLT outlet.

Visitors to VLT outlets have to identify themselves with a valid photo ID and a terminal can only be operated with a personalized player card. Player spending on VLTs is recorded by the operator due the required use of a personalized player card. After gambling for two hours, the gambler must have a 'cool down' phase. To restrict the loss, a maximum stake of $€ 10$ per game has been established.

The VLT licensee must implement a gradual player protection warning system which depends on the number of visits and money spent. Based on that, the VLT licensee can enquire about a players' personal income with a third party. The VLT licensee can then demand a personal conversation and, for example, negotiate a limited visiting frequency or ban players permanently or temporarily, if it can be shown that the players' financial resources are not sufficient. At win2day, gamblers can deposit a maximum of $€ 800$ a week. win2day also uses the player tracking tool mentor ${ }^{7}$ which analyses player behaviour and provides personalized feedback to players to improve their awareness.

\section{Federal state law}

Each Austrian federal state can decide for itself whether it permits slot machine establishments or not. Federal regulations may be more restrictive, but all regulations have to conform to the countrywide minimum standards outlined above. In contrast to VLTs, which are regulated at the national level, the computation of slot machine analytics occurs on the machine itself rather than a central computing centre. This is the main difference between federally-regulated slot machines and nationally-regulated VLTs. To date, five (of the nine) federal states have awarded slot machine licenses to several different operators, some of which operate in more than one federal state. The federal state law distinguishes between gambling outlets with 10-50 slot machines and gambling outlets which can have a maximum of three slot machines (e.g., small shops, restaurants, bars, etc.). Special regulation applies to

\footnotetext{
7 Auer, M. \& Griffiths, M.D. (2015). The use of personalized behavioral feedback for problematic online gamblers: An empirical study. Frontiers in Psychology, 6, 1406
} 
both categories $(\$ 5 \mathrm{GSpG})$. All slot machines have to be connected to the Austrian computing centre. In both gambling venues, visitors of full age (18+ years) are allowed to gamble.

Licensees with 10-50 slot machines must implement a player protection warning system. Gambling venues with three or fewer slot machines are required to issue a personalized player card to monitor the maximum allowed playing duration of three hours over a 24-hour period. For bigger outlets with 10-50 slot machines, after two hours, the gambler must have a 'cool down' phase. For smaller outlets, the maximum bet must not exceed $€ 1$, the minimum game duration must not be less than two seconds, and the maximum win must not be higher than $€ 1,000$. For outlets with $10-50$ slot machines, the maximum bet must not exceed $€ 10$, the minimum game duration must not be less than one second, and the maximum win must not be higher than $€ 10,000$. Large jackpots are not allowed in small gambling venues. The GSpG (§5) specifically requires an individual to use a player card in small shops with up to three slot machines. However, if biometric identification methods are used, a physical player card does not have to be used because these methods are viewed as equivalent in its functionality to the player card. For example, when players first come to play on a slot machine, their fingerprints are digitally scanned along with their names, addresses, and whatever other information is required. The slot machines have a fingerprint sensor so whenever players want to gamble on a slot machine they simply have to put their finger on the sensor instead of using a player card. The slot machine knows the player and their gambling data are recorded and matched with the players' ID. This could also be done with facial recognition, iris scans, or anything else that provides a unique biometric marker of the gambler.

\section{Concluding remarks}

To the best of the authors' knowledge, it appears that all federal slot machine licensees have introduced mandatory player cards or a form of biometric identification method. Not only does this allow gaming operators to monitor the gambling behaviour of their clientele; but, it means that players are not allowed to play several machines at once as well as having good use in cases of (voluntary self-)exclusion. As far as the authors are aware, Austria is one of only three countries worldwide that have introduced mandatory player cards or a comparable biometric identification option to monitor player behaviour and to use this information for player protection. 
In Sweden and Norway, all players who gamble on gambling products offered by Svenska Spel (the Swedish monopoly gambling operator) and Norsk Tipping (the Norwegian monopoly gambling operator) have to use a player card. The use of player cards demonstrates a high level commitment to player protection and over time, it is expected that other countries will follow such initiatives as a way of demonstrating to the general public within those jurisdictions that gambling operators have a duty of care to its clientele in protecting them from the potential dangers of excessive gambling. Arguably, Sweden and Norway have even greater levels of player protection because their player cards are used for all products within their gambling portfolios whereas Austria's player cards are only required for slot machine gambling. There are responsible gambling experts based in Austria who would like the Austrian player card to be similar to that used in Sweden and Norway, and to be applied across all gambling offers and not just slot machines. It should also be noted that mandatory use of player cards does not prevent players from accessing other gambling operators' products in other jurisdictions and countries (particularly because online gambling is by definition a global activity), but having a culture of responsible gambling within a particular country may lead players in those countries to gamble more responsibly when using products that do not require the use of a player card.

The gambling laws introduced in Austria clearly differentiate between national and federal gambling regulation with little in the way of ambiguity. Federal regulators have the power to impose even stricter regulations on gambling if they so wish, but no federal regulator can do anything less than what is stated in the national gambling laws. The Austrian gambling laws demonstrate that Austrian legislators have been proactive in the field of responsible gambling and player protection. The country is also among the minority that have legalised online gambling. Interestingly, online gambling regulation in Austria is inextricably linked to the national lottery operators. This may be because the national lottery operator in Austria arguably provides the most robust responsible gambling infrastructure of all Austrian gaming operators. Gambling legislators in other countries could learn much from what has been introduced in countries such as Austria, Sweden, and Norway in terms of responsible gambling, social responsibility, player protection, and harm minimisation. If countries are serious about minimising gambling harm and wish to keep problem gambling to an absolute minimum, the use of mandatory player cards appears to be one part of a bigger solution. While technological advances can be used to exploit gamblers even more so than has 
happened in the past, they can equally be used to harness and protect gamblers from playing beyond their means (in both time and money). 